Original

\title{
Resonance frequency analysis of miniscrew implant stability
}

\author{
Yu-Chuan Tseng1,2), Chin-Yun Pan 1,2), Pao-Hsin Liu'3), Yi-Hsin Yang4,5), \\ Hong-Po Chang ${ }^{1,6)}$, and Chun-Ming Chen ${ }^{1,7)}$
}

\author{
1)School of Dentistry and Graduate Program of Dental Science, Kaohsiung Medical University, \\ Kaohsiung, Taiwan, ROC \\ 2)Department of Orthodontics, Dental Clinics, Kaohsiung Medical University Hospital, Kaohsiung, Taiwan, \\ ROC \\ 3)Department of Biomedical Engineering, College of Medicine, I-Shou University, Kaohsiung, Taiwan, ROC \\ 4)Medical Informatics and Statistics Center, Research and Development Office, Kaohsiung Medical University, \\ Kaohsiung, Taiwan, ROC \\ 5)Division of Medical Statistics and Bioinformatics, Department of Medical Research, \\ Kaohsiung Medical University Hospital, Kaohsiung, Taiwan, ROC \\ 6)Department of Dentistry (Orthodontics), Kaohsiung Municipal Hsiao-Kang Hospital, \\ Kaohsiung, Taiwan, ROC \\ 7)Department of Oral and Maxillofacial Surgery, Dental Clinics, Kaohsiung Medical University Hospital, \\ Kaohsiung, Taiwan, ROC
}

(Received August 16, 2016; Accepted February 22, 2017)

\begin{abstract}
This study used resonance frequency (RF) analysis to assess miniscrew implant (MSI) stability during wound healing in a sample of 68 patients (41 women, 27 men; mean age, 27.7 years). The 104 MSIs included 66 placements in the buccal shelf (BS; $2.0 \times$ $12 \mathrm{~mm}$ ) and 38 placements in interradicular (IR; 1.5 $\times 8 \mathrm{~mm}$ ) sites. Thirteen $(12.5 \%)$ of the MSIs failed. A new RF detection device was used to measure RF at baseline (T0) and at 3 (T1), 6 (T2), 9 (T3), 12 (T4), and 15 (T5) weeks after placement. A linear mixed-effects model was fitted to change in RF values. As compared with the BS group, the IR group had significantly lower RF values on the right side from $\mathrm{T} 0$ through $\mathrm{T} 4$ and on the left side from $\mathrm{T} 0$ through $\mathrm{T} 2$. Insertion site and time of visit were significantly associated with RF value. The effects of time of visit significantly differed
\end{abstract}

Correspondence to Dr. Hong-Po Chang, School of Dentistry and Graduate Program of Dental Science, Kaohsiung Medical University, Shih-Chuan 1st Road, Kaohsiung 80708, Taiwan Fax:+886-7-3210637 E-mail: chang610004@gmail.com

Color figures can be viewed in the online issue at J-STAGE. doi.org/10.2334/josnusd.16-0613

DN/JST.JSTAGE/josnusd/16-0613 between the BS and IR sites. Starting from T0, the MSIs placed at both sites had significantly lower RF values at all intervals, except for T0-T1. Future studies should examine how the present clinical protocols can optimize timing of MSI loading to maximize the success rate.

Keywords: miniscrew; implant stability; mandible; resonance frequency analysis.

\section{Introduction}

Miniscrew implants (MSIs) are established skeletal anchorage devices routinely used in orthodontic practice (1). Anchorage stability is a key factor in the success of orthodontic treatment.

Implant stability is usually divided into two stages. Primary stability results from mechanical engagement with cortical bone (implant stability reached during implant placement). Secondary stability develops from regeneration and remodeling of bone and tissue around the implant after insertion and is affected by primary stability, bone formation, and remodeling (implant stability after healing). The combined effects of primary 

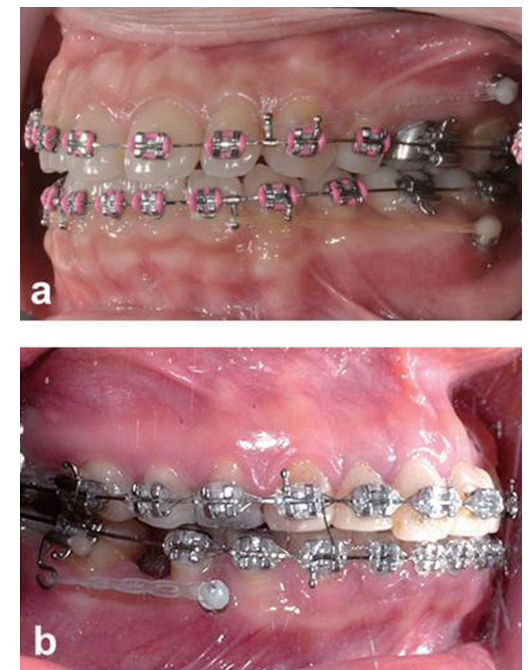

Fig. 1 Implantation sites. (a) The miniscrew implant was placed in the buccal shelf. (b) The miniscrew implant was placed between the lower first premolar and the second premolar on the buccal side.

and secondary stability determine the total stability of an implant (2). After an implant placement, biological factors that affect secondary stability gradually become more important than mechanical factors that affect primary stability. Wound healing is characterized by the transition from primary mechanical stability, which is provided by local bone quality and quantity, to biological stability, which is provided by newly formed bone (3). During the healing phase, MSI stability is affected by changes caused by the remodeling processes (4).

In 2000, the Osstell device (Integration Diagnostics $\mathrm{AB}$, Göteborg, Sweden) was introduced for monitoring dental implant status. When a detection device is used for resonance frequency (RF) analysis, stable coupling between the SmartPeg transducer and implant is needed. A transducer suitable for the size and structure of a particular MSI may be difficult to obtain. In addition, the head of the MSI must be modified to fit the Osstell transducer (5). For clinical use, the Implomates (BioTech One, Inc., Taipei, Taiwan) is more convenient than the Osstell for detecting RF values of MSI without modification.

This study used the Implomates, a new RF detection device, to monitor MSI stability. High and low stability were defined as high and low RF values, respectively (6), and RF measurements were used to evaluate MSI stability during wound healing.

\section{Materials and Methods}

Data from patients whose orthodontic treatment plan called for MSI insertion into the buccal shelf (BS) or interradicular (IR) sites of the mandible were analyzed

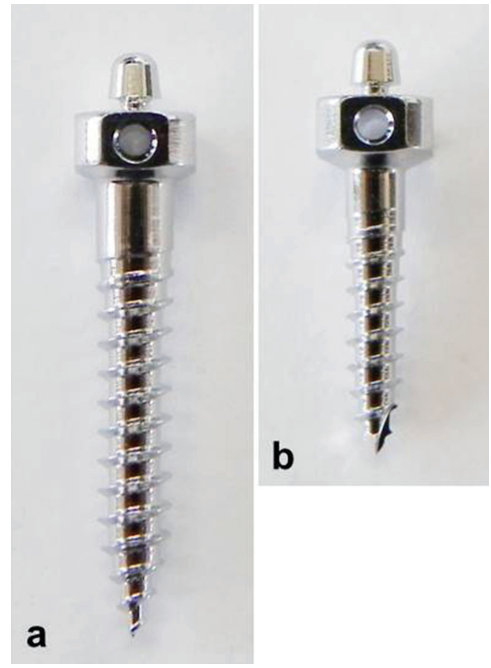

Fig. 2 Miniscrew implants. (a) Stainless steel miniscrew implant (diameter, $2.0 \mathrm{~mm}$; length, $12 \mathrm{~mm}$ ). (b) Stainless steel miniscrew implant (diameter, $1.5 \mathrm{~mm}$; length, $8 \mathrm{~mm}$ ).

(Fig. 1). Written informed consent was obtained from all patients before the study was begun. The consent procedure and treatment protocol were approved by the Institutional Review Board of Kaohsiung Medical University Hospital (KMUH-IRB-990363). This prospective study was performed in accordance with the Declaration of Helsinki guidelines for experiments involving human subjects.

The mean age of the participants (41 women, 27 men) at the insertion stage was 27.7 years (SD, 5.13 years; range, 18-37 years). After MSI placement for 3 weeks, elastomeric chains were used to apply orthodontic force (150-200 grams) to MSIs. Of the 104 stainless steel MSIs (Bio-Ray, Syntec Scientific Corp., Taipei, Taiwan) inserted, 66 were inserted at BS sites (diameter, $2 \mathrm{~mm}$; length, $12 \mathrm{~mm}$ ) as anchorage for distalization of anterior teeth and 38 were inserted at IR sites (diameter, $1.5 \mathrm{~mm}$; length, $8 \mathrm{~mm}$ ) as anchorage for mesialization of posterior teeth (Figs. 1, 2). The MSIs were self-drilling and were inserted with a hand driver.

Bisecting-angle dental radiographs and orthoradial projection technique were used for evaluation (7) and showed that MSIs for successful cases were completely separate from the root and did not touch the lamina dura or the root at implantation or after orthodontic treatment.

\section{Resonance frequency analysis}

We measured the RF of inserted MSIs at baseline (T0) and at 3 (T1), 6 (T2), 9 (T3), 12 (T4), and 15 weeks (T5) after MSI placement. All measurements were made with an RF analyzer (Implomates) (Fig. 3). Each MSI was measured three times, and the mean value was recorded. 

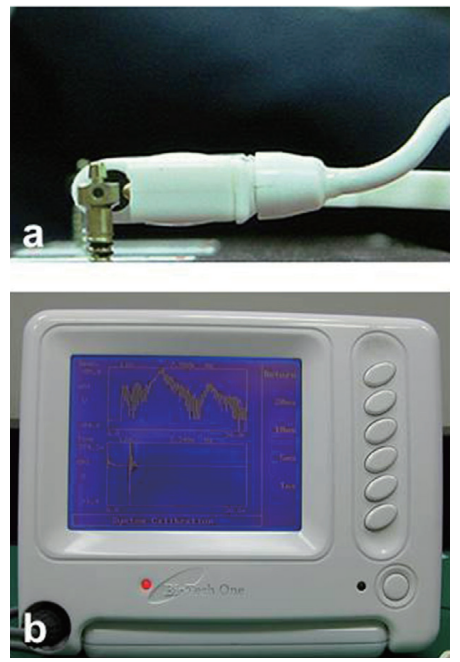

Fig. 3 The Implomates resonance frequency analyzer used in the study. The device uses an impact force to excite resonance in the miniscrew implant. (a) The impact force is provided by a small electrically driven impact rod inside the transducer. (b) The received response signal is then transferred to a computer for frequency spectrum analysis.

Table 1 Characteristics of the participants

\begin{tabular}{lcc}
\hline Characteristic & Buccal shelf & Interradicular site \\
\hline Sex & & \\
$\quad$ Female & 52 & 18 \\
$\quad$ Male & 14 & 20 \\
Age \pm SD (years) & & \\
$\quad$ Right side & $26.76 \pm 4.58$ & $28.34 \pm 6.43$ \\
$\quad$ Left side & $26.47 \pm 4.50$ & $28.74 \pm 5.37$ \\
Side & 34 & \\
$\quad$ Total number on right side & 32 & 17 \\
$\quad$ Total number on left side & 2 & 3 \\
$\quad$ Failures on right side & 4 & 4 \\
Failures on left side & & \\
Visit (no. of failures) & T5 (2) & T5 (3) \\
Visit, failure on right side & T4 (1), T5 (3) & T3 (1), T5 (3) \\
$\quad$ Visit, failure on left side & & \\
$\quad$ & &
\end{tabular}

\section{Statistical analysis}

Change in RF value at each visit was fitted to a linear mixed-effects model, which considered measurements taken at different time points and from different MSI sites in the same patient as random effects. Covariates, i.e., the fixed effects, included sex, age, insertion side (right or left), insertion site (BS or IR site), time of visit (T0-T5), and all possible interactions between visits. Adjusted mean RF values were estimated at each visit, and the difference between two insertion sites was tested for each patient. Statistical significance was defined as a $P$ value of $<0.05$. All data analysis was performed with
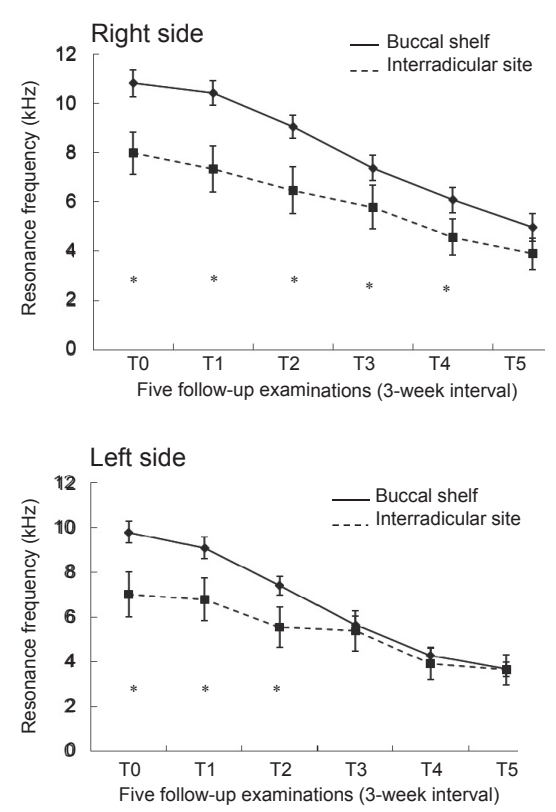

Fig. 4 Resonance frequency values for the two insertion sites at five follow-up examinations separated by 3 -week intervals. Whiskers indicate $95 \%$ confidence intervals. *Significant difference between groups $(P<0.05)$.

Table 2 Results of statistical analysis of change in resonance frequency, a linear mixed-effects model

\begin{tabular}{|c|c|c|c|c|}
\hline & \multicolumn{2}{|c|}{$P$ value } \\
\hline \multicolumn{5}{|c|}{ Variable } \\
\hline \multicolumn{3}{|c|}{ Sex (male vs female) } & & 0.33 \\
\hline \multicolumn{3}{|c|}{ Age (at insertion) } & & 0.19 \\
\hline \multicolumn{3}{|c|}{ Side (right vs left) } & & 0.49 \\
\hline \multicolumn{3}{|c|}{ Site (buccal shelf vs interradicular) } & & 0.003 \\
\hline \multicolumn{3}{|c|}{ Visit (T0, T1, T2, T3, T4, T5) } & & $<0.0001$ \\
\hline \multicolumn{5}{|c|}{ Test } \\
\hline \multicolumn{3}{|c|}{ Site vs visit } & & $<0.0001$ \\
\hline & \multicolumn{2}{|c|}{ Buccal shelf } & \multicolumn{2}{|c|}{ Interradicular site } \\
\hline & Adjusted mean & $95 \% \mathrm{CI}$ & Adjusted mean & $95 \% \mathrm{CI}$ \\
\hline T0 & 11.0 & $10.3,11.6$ & 8.2 & $7.4,9.0$ \\
\hline T1 & 10.5 & $9.8,11.1$ & 7.6 & $6.8,8.4$ \\
\hline $\mathrm{T} 2$ & 9.0 & $8.4,9.7$ & 6.7 & $5.9,7.5$ \\
\hline T3 & 7.3 & $6.6,8.0$ & 5.8 & $5.0,6.6$ \\
\hline T4 & 6.0 & $5.4,6.7$ & 4.7 & $3.9,5.5$ \\
\hline T5 & 4.9 & $4.2,5.6$ & 3.7 & $2.9,4.4$ \\
\hline
\end{tabular}

the SAS software package (Version 9, SAS Institute Inc., Cary, NC, USA).

\section{Results}

Of the 104 MSI placements analyzed in this study, 13 $(12.5 \%)$ were failed. Table 1 shows the number of failures observed at each time point: one failure occurred at $\mathrm{T} 3$, one at $\mathrm{T} 4$, and 11 at $\mathrm{T} 5$.

Figure 4 shows change in RF values for the MSIs. On the right side, RF values were significantly lower in the IR group than in the BS group at T0, T1, T2, T3, and T4 (all $P<0.05$ ) but did not significantly differ between 
Table 3 Resonance frequency values for T0, T1, T2, T3, T4, and T5

\begin{tabular}{|c|c|c|c|c|c|c|}
\hline & \multicolumn{3}{|c|}{ Buccal shelf } & \multicolumn{3}{|c|}{ Interradicular site } \\
\hline & Adjusted mean & $95 \% \mathrm{CI}$ & $P$ value & Adjusted mean & $95 \% \mathrm{CI}$ & $P$ value \\
\hline T0-T1 & 0.46 & $-0.07,0.99$ & 0.09 & 0.64 & $0.01,1.26$ & 0.05 \\
\hline T0-T2 & 1.94 & $1.40,2.49$ & $<0.0001$ & 1.47 & $0.83,2.11$ & $<0.0001$ \\
\hline T0-T3 & 3.74 & $3.19,4.29$ & $<0.0001$ & 2.28 & $1.63,2.93$ & $<0.0001$ \\
\hline T0-T4 & 5.12 & $4.56,5.67$ & $<0.0001$ & 3.42 & $2.78,4.06$ & $<0.0001$ \\
\hline T0-T5 & 6.19 & $5.63,6.74$ & $<0.0001$ & 4.43 & $3.80,5.07$ & $<0.0001$ \\
\hline $\mathrm{T} 1-\mathrm{T} 2$ & 1.51 & $1.23,1.78$ & $<0.0001$ & 0.82 & $0.47,1.18$ & $<0.0001$ \\
\hline $\mathrm{T} 2-\mathrm{T} 3$ & 1.82 & $1.54,2.10$ & $<0.0001$ & 0.81 & $0.45,1.17$ & $<0.0001$ \\
\hline T3-T4 & 1.39 & $1.11,1.67$ & $<0.0001$ & 1.13 & $0.77,1.49$ & $<0.0001$ \\
\hline T4-T5 & 1.06 & $0.77,1.35$ & $<0.0001$ & 1.02 & $0.63,1.41$ & $<0.0001$ \\
\hline
\end{tabular}

these groups at $\mathrm{T} 5(P>0.05)$. On the left side, $\mathrm{RF}$ values were significantly lower in the IR group than in the BS group at T0, T1, and T2 $(P<0.05)$ but did not significantly differ at T3 through T5 $(P>0.05)$.

Table 2 shows adjusted mean RF values and 95\% confidence intervals for MSIs at the BS and IR sites. Statistical analysis showed that insertion site and time of visit were significantly associated with RF value $(P$ $=0.003$ and $P<0.0001$, respectively). A formal test for site-by-visit interaction suggested that the effects of the visit significantly differed between the BS and IR sites $(P<0.0001)$. RF values were not significantly affected by sex, age, or insertion side $(P>0.05)$. RF values of MSIs were significantly lower at the BS and IR sites at visits after T0 and between visits $(P<0.0001)$. The only exception was the interval T0-T1 $(P>0.05)$ (Table 3$)$.

\section{Discussion}

Accurate measurement orthodontic MSI stability is a continuing challenge. This study compared the effects of different materials on the primary stability of MSIs by using a relatively new RF analyzer, the Implomates device, which was used in a previous study of artificial bones (8). To our knowledge, this is the first clinical study to use the Implomates to evaluate change in MSI stability during the wound healing.

$\mathrm{RF}$ analysis is the most promising method for noninvasive diagnostic assessment of dental implant/MSI stability. The Osstell device utilizes the basic principles of the harmonic response method. The Osstell is used by screwing the transducer into the dental implant with a torque of $10 \mathrm{Ncm}$, which is almost half the force used for implant insertion and may result in microdamage (9). The specifications and selection of transducers used for dental implants are detailed in the SmartPeg Reference Guidance (Osstell). A transducer suitable for the size and structure of a particular MSI may be difficult to obtain. In addition, the MSI head must be modified to fit the Osstell transducer (5). When the device is used for RF analysis, stable coupling between the SmartPeg transducer and the implant is necessary. Experiments using beagles showed that failure usually occurred when the transducer was screwed into the modified head of the MSI or when it was unscrewed after recordings were obtained (10). RF analysis can also be performed by using the Implomates device for impulse force triggering. The Implomates transducer minimizes contact and avoids torque. Additionally, no installation or disassembly is required. The device uses impact force to excite resonance in the miniscrew implant. Impact force is provided by a small electrically driven rod inside the transducer (Fig. 3a). The received response signal is then transferred to a computer for frequency spectrum analysis (Fig. 3b). A dental implant study reported that Implomates RF values and implant stability quotient (ISQ) values yielded by the Osstell device exhibited a strong positive linear correlation $(r=0.991, P<0.01)(6)$.

The success rate for orthodontic MSIs used as temporary anchorage range was $57 \%$ to $95.3 \%$ and usually approximated $84 \%$ (11). The present overall success rate was $87.5 \%$. Motoyoshi et al. (12) reported MSI success rates of $88.9 \%$ to $100.0 \%$ in adults, $55.6 \%$ to $71.4 \%$ in early-load adolescents, and $90.9 \%$ to $100.0 \%$ in late-load adolescents. The titanium alloy MSIs used in their study were inserted into alveolar bones, and orthodontic forces were loaded after implant placement for 2 to 4 weeks (mean, 2.6 weeks) in early-load adolescents, for 3 months or longer (mean, 13.2 weeks) in late-load adolescents, and for 2 to 4 weeks (mean, 2.2 weeks) in adults.

Two key determinants of primary MSI stability are bone quality and quantity (13). Cortical bone quantity and quality affect long-term stability. Stationary anchorage failure is often caused by low bone density or inadequate cortical bone thickness (14). Cortical bone thickness is important in the success of an MSI because insufficient cortical bone thickness often causes inadequate primary 
stability. If primary stability is not achieved at insertion, the MSI may loosen during orthodontic treatment $(1,11)$. Motoyoshi et al. $(12,15)$ reported that a cortical bone thickness greater than $1 \mathrm{~mm}$ was needed for adequate primary stability and achieved acceptable success rates for MSI placements. In most cases, altering the MSI insertion angle can compensate for insufficient cortical bone thickness.

Primary stability depends mainly on the mechanical characteristics of the original bone (its local quality and quantity), implant characteristics (geometry, diameter, length, and surface), and surgical technique used. Implant size is significantly associated with RF value. Increased insertion depth yields a high RF value, which increases primary implant stability (8). Therefore, MSI insertion depth should be maximized to obtain maximum stability. The primary stability of an MSI can also be increased by increasing implant length. Conversely, as the exposed length increases, the RF value and the primary stability decrease. In this study, the stainless steel MSIs inserted into the BS were $12 \mathrm{~mm}$ in length and $2 \mathrm{~mm}$ in diameter, whereas those inserted into the IR sites were $8 \mathrm{~mm}$ in length and $1.5 \mathrm{~mm}$ in diameter.

After MSI placement, primary mechanical stability is gradually replaced by biological secondary stability. The transition from mechanical stability to biological stability provided by newly formed bone occurs early during wound healing (3). Therefore, a critical interval in the healing process is the period during which osteoclastic activity has decreased the initial mechanical stability of the implant but new bone formation is still insufficient to maintain implant stability. Therefore, the healing period should be long enough to achieve sufficient secondary stability before loading (16).

Misch et al. (17) proposed four bone density classifications, D1-D4, based on Hounsfield units (HU): D1 for $\mathrm{HU}$ values greater than 1,250, D2 for $\mathrm{HU}$ values of 850 to $1,250 \mathrm{HU}, \mathrm{D} 3$ for $\mathrm{HU}$ values of 350 to 850 , and $\mathrm{D} 4$ for $\mathrm{HU}$ values of 150 to 350 . Dense cortical bone in the BS is classified as D1. Porous cortical bone with coarse trabeculae in the posterior mandibular IR site is D2. Thin, porous cortical bone with fine trabeculae, in some posterior mandibular IR sites, is D3. Fine trabecular bone, which is mainly found in the maxillary tuberosity, is D4. Miniscrew implant placements in D4 bone have high failure rates and are not recommended $(18,19)$.

In this study, RF values were higher at BS sites than at IR sites. However, the differences were statistically significant only at placement and at 3, 6, 9, and 12 weeks, on the right side, and at placement and at 3 and 6 weeks, on the left side. The differences were not significant at 15 weeks, on the right side, or at 9, 12, or 15 weeks, on the left side. Both the BS and IR sites had significantly lower RF values from week 3 through week 15 (Fig. 4). Ure et al. (10) reported similar results in an animal study of MSI placements in maxillae. ISQ values decreased during the first 3 weeks after placement and increased from week 3 to week 5. At the end of the healing period, however, ISQ had not returned to the initial values.

MSIs in the mandibular BS have been proposed as a reliable source of extra-alveolar anchorage for retracting the entire mandibular arch, to correct severe crowding, protrusion, and skeletal malocclusion. Placing an MSI in an extra-alveolar site like the mandibular BS permits the use of larger-diameter screws that can be inserted parallel to the axial inclination of molars and will not interfere with tooth roots (20). Root contact during placement of miniscrews in IR sites in the alveolar process for orthodontic anchorage significantly increases the risk of implant failure (21). Even when IR placement is placement accurate, the MSI may accidentally touch an adjacent root during mesialization of posterior dentition. The proximity of an MSI to the adjacent root is an important risk factor in the failure of miniscrew anchorage. Therefore, an MSI placement that avoids root proximity is important in the stability of miniscrews for orthodontic anchorage. However, the associations of root proximity and root contact with RF values were not examined in this study, and should be investigated in future.

Bone-implant adaptation is typically measured by assessing bone-to-implant contact ratio (implant surface in contact with bone/total implant surface) (22). A boneto-implant contact ratio as low as $5 \%$ was sufficiently resistant to orthodontic loads (16). Partial osseointegration of titanium-alloy MSIs is a distinct advantage in orthodontic applications because it provides effective anchorage and easy insertion and removal (23). Unlike titanium alloy, stainless steel bone screws tend to develop a fibrous tissue interface between the screw and bone (24). We speculate that fibrous tissues that develop around stainless steel screw threads increase risk of implant failure, by reducing the bone-to-implant contact.

Over time, differences in bone-to-implant contact can result from fibrous tissue formation caused by contact with stainless steel. Future studies should compare longterm stability of orthodontic MSIs composed of titanium alloy and those made of stainless steel. In addition, the present methods and clinical protocols should be further investigated, to determine the optimal timing of MSI loading, as indicated by skeletal anchorage success rates. 


\section{Acknowledgments}

The authors are grateful to the Kaohsiung Medical University Hospital, Taiwan, for financial support of this study (KMUH999M30).

\section{Conflict of interest}

None declared.

\section{References}

1. Chang HP, Tseng YC (2014) Miniscrew implant applications in contemporary orthodontics. Kaohsiung J Med Sci 30, 111-115.

2. Raghavendra S, Wood MC, Taylor TD (2005) Early wound healing around endosseous implants: a review of the literature. Int J Oral Maxillofac Implants 20, 425-431.

3. Berglundh T, Abrahamsson I, Lang NP, Lindhe J (2003) De novo alveolar bone formation adjacent to endosseous implants. Clin Oral Implants Res 14, 251-262.

4. Gedrange T, Proff P, Bayerlein T, Landsberger P, Dietze S, Fanghänel J (2006) Histological and fluorescence microscopic examination of the bone/implant interface in orthodontic miniscrews (Mondeal ${ }^{\circledR}$ ). Folia Morphol (Warsz) 65, 70-71.

5. Nienkemper M, Wilmes B, Panayotidis A, Pauls A, Golubovic V, Schwarz F et al. (2013) Measurement of mini-implant stability using resonance frequency analysis. Angle Orthod 83, 230-238.

6. Chang WJ, Lee SY, Wu CC, Lin CT, Abiko Y, Yamamichi N et al. (2007) A newly designed resonance frequency analysis device for dental implant stability detection. Dent Mater J 26, 665-671.

7. Kuroda S, Yamada K, Deguchi T, Hashimoto T, Kyung HM, Takano-Yamamoto T (2007) Root proximity is a major factor for screw failure in orthodontic anchorage. Am J Orthod Dentofacial Orthop 131, S68-73.

8. Pan CY, Chou ST, Tseng YC, Yang YH, Wu CY, Lan TH et al. (2012) Influence of different implant materials on the primary stability of orthodontic mini-implants. Kaohsiung J Med Sci 28, 673-678.

9. Tonetti MS, Schmid J (1994) Pathogenesis of implant failures. Periodontol 2000 4, 127-138.

10. Ure DS, Oliver DR, Kim KB, Melo AC, Buschang PH (2011) Stability changes of miniscrew implants over time: a pilot resonance frequency analysis. Angle Orthod 81, 994-1000.

11. Schätzle M, Männchen R, Zwahlen M, Lang NP (2009)
Survival and failure rates of orthodontic temporary anchorage devices: a systematic review. Clin Oral Implants Res 20, 1351-1359.

12. Motoyoshi M, Matsuoka M, Shimizu N (2007) Application of orthodontic mini-implants in adolescents. Int J Oral Maxillofac Surg 36, 695-699.

13. Kravitz ND, Kusnoto B (2007) Risks and complications of orthodontic miniscrews. Am J Orthod Dentofacial Orthop 131, S43-51.

14. Melsen B, Verna C (2005) Miniscrew implants: the Aarhus anchorage system. Semin Orthod 11, 24-31.

15. Motoyoshi M, Inaba M, Ono A, Ueno S, Shimizu N (2009) The effect of cortical bone thickness on the stability of orthodontic mini-implants and on the stress distribution in surrounding bone. Int J Oral Maxillofac Surg 38, 13-18.

16. Deguchi T, Takano-Yamamoto T, Kanomi R, Hartsfield JK Jr, Roberts WE, Garetto LP (2003) The use of small titanium screws for orthodontic anchorage. J Dent Res 82, 377-381.

17. Misch CE, Dietsh-Misch F, Hoar J, Beck G, Hazen R, Misch CM (1999) A bone quality-based implant system: first year of prosthetic loading. J Oral Implantol 25, 185-197.

18. Kravitz ND, Kusnoto B, Tsay TP, Hohlt WF (2007) The use of temporary anchorage devices for molar intrusion. J Am Dent Assoc 138, 56-64.

19. Chun YS, Lim WH (2009) Bone density at interradicular sites: implications for orthodontic mini-implant placement. Orthod Craniofac Res 12, 25-32.

20. Chang C, Liu SS, Roberts WE (2015) Primary failure rate for 1680 extra-alveolar mandibular buccal shelf mini-screws placed in movable mucosa or attached gingiva. Angle Orthod 85, 905-910.

21. Chen YH, Chang HH, Chen YJ, Lee D, Chiang HH, Yao CC (2008) Root contact during insertion of miniscrews for orthodontic anchorage increases the failure rate: an animal study. Clin Oral Implants Res 19, 99-106.

22. Uemura M, Motoyoshi M, Yano S, Sakaguchi M, Igarashi Y, Shimizu N (2012) Orthodontic mini-implant stability and the ratio of pilot hole implant diameter. Eur J Orthod 34, 52-56.

23. VandeVannet B, Sabzevar MM, Wehrbein H, Asscherickx K (2007) Osseointegration of miniscrews: a histomorphometric evaluation. Eur J Orthod 29, 437-442.

24. Albrektsson T, Hansson HA (1986) An ultrastructural characterization of the interface between bone and sputtered titanium or stainless steel surfaces. Biomaterials 7, 201-205. 